\title{
Suicide rate 22 years after parasuicide: cohort study
}

\author{
Gary R Jenkins, Robert Hale, Maria Papanastassiou, Michael J Crawford, Peter Tyrer
}

The rate of suicide for people who have had an episode of parasuicide is 100 times higher in the year following the episode than that of the general population. ${ }^{1}$ Providing a high standard of care to patients who deliberately harm themselves could help to reduce this rate. ${ }^{2}$ Long term follow up studies show that the increased rate of suicide persists. ${ }^{3}$ However, the long term risk of suicide in patients in the United Kingdom is uncertain. We traced a consecutive sample of patients 22 years after they presented to a central London teaching hospital after an episode of parasuicide in the late 1970s.

\section{Method and results}

From May 1977 to March 1980 one of us (RH) collected demographic and clinical data on a consecutive sample of weekday ward referrals to psychiatric services after patients had an episode of parasuicide. Twenty two years later we attempted to trace the patients, using data from the Office for National Statistics. In accordance with previous studies we combined deaths that were recorded as "suicide" with deaths in which data on the death certificate suggested that the cause of death was "probable suicide." Cause of death was attributed to probable suicide by two raters (GRJ and MP) independently, and there was full agreement between these two sets of ratings. Data were analysed by using the statistical software package SPSS (version 9.0). The rate of suicide during the period of follow up was calculated and then illustrated by using survival curves. We examined longitudinal associations between subsequent suicide and potential explanatory factors by using Cox regression. Hazard ratios with $95 \%$ confidence intervals are presented.

A total of 223 patients were interviewed between May 1977 and March 1980. Most (154, 69\%) were women, with a mean age of 32 years. Basic demographic details required by the Office for National Statistics to trace patients had been recorded for 180 patients, 40 of whom could not be traced. The characteristics of the 140 who were traced and the 83 not traced were similar. However, patients who were not traced were more likely to come from ethnic minorities and more likely to have consumed alcohol at the time of parasuicide than patients who were traced (25\% v 9\%, difference in proportions 18\% (95\% confidence interval $12 \%$ to $38 \%$ ) and $28 \%$ v $16 \%, 12 \%$ ( $2 \%$ to $24 \%$ ), respectively). The remaining 140 were followed until July 2000 (mean follow up 21.75 years, range 20.3-23.1 years). During this period 25 (18\%) died. Examination of death certificates revealed three suicides and nine probable suicides (four were recorded as open verdict and five as accidental death). The overall rate of probable suicide was 4.3 (2.4 to 7.7) per 1000 per year.

The figure illustrates the rate of survival from suicide plus probable suicide during the period of fol-

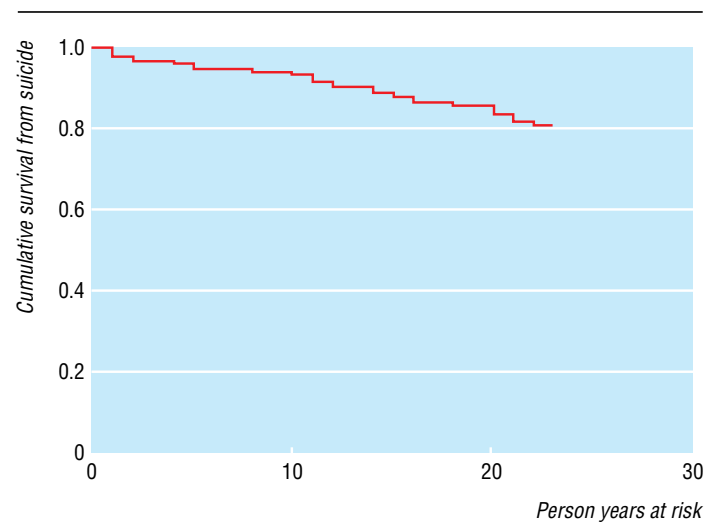

Survival from suicide of 140 patients followed over 22 years after parasuicide. Deaths from other causes were censored

low up. The rate was $5.9 / 1000 /$ year in the first five years, 0/1000/year between five and 10 years, 4.7/1000/year between 10 and 15 years, 5.0/1000/ year between 15 and 20 years, and 6.8/1000/year in the final three years. The rate did not decline with time $\left(\chi^{2}=0.35, \mathrm{df}=1, \mathrm{P}=0.55\right.$ for trend $)$.

\section{Comment}

The risk of suicide for people with a history of parasuicide persists over many years. Our findings are based on $63 \%$ of the original sample (with the people being at a higher risk of repetition, given that it is an inpatient sample); we were unable to estimate the rate of suicide in patients not traced. Clinicians are encouraged to pay particular attention to the management of patients immediately after an episode of parasuicide. ${ }^{5}$ Previous deliberate self harm remains a potent risk factor for subsequent suicide, even if it occurred many years ago.

We thank the Office for National Statistics for provision of data. Contributors: RH collected the initial data and proposed the idea of the study; GRJ initiated the follow up. GRJ and MP drafted the protocol, gathered data from the ONS, and wrote the paper. MJC performed the data analysis and supervised editing of the paper, and PT supervised the overall process. GRJ is the guarantor.

Funding: Nicola Pigott Memorial Fund.

Competing interests: None declared.

1 Greer S, Bagley C. Effect of psychiatric intervention in attempted suicide: a controlled study BMJ 1971;i:310-2

2 Royal College of Psychiatrists. Guidance on the management of deliberate self-harm Psychiatr Bull 1994;7:210-2.

3 De Moore GM, Robertson AR. Suicide in the 18 years after deliberate self-harm. A prospective study. BrJ Psychiatry 1996;169:489-94.

4 Hawton K, Fagg J. Suicide, and other causes of death, following attempted suicide. Br J Psychiatry 1988;152:59-66.

5 Crawford MJ, Wessely S. Does initial management affect the rate of repetition of deliberate self harm? Cohort study. BMJ 1998;317:985.
Editorial by Runeson

Department of Psychiatry, East Ham Memorial Hospital, London E7 8QR

Gary R Jenkins consultant psychiatrist

Portman Clinic,

Tavistock and

Portman NHS

Trust, London

NW3 5NA

Robert Hale

consultant

psychotherapist

Department of

Public Mental

Health, Faculty of

Medicine, Imperial

College of Science,

Technology and

Medicine, London

W2 1PD

Maria

Papanastassiou

specialist registrar in psychiatry

Michael J Crawford

senior lecturer in

psychiatry

Peter Tyrer

professor of

community psychiatry

Correspondence to: G R Jenkins

Newnham Centre

for Mental Health,

Glen Road, London

E13 8SP

gary.jenkins@

elcmht.nhs.uk

BMJ 2002;325:1155 